\title{
Effect of Indium addition in U-Zr Metallic Fuel on Lanthanide Migration
}

Yeon Soo Kim *, T. Wiencek, E. O’Hare, J. Fortner, A. Wright 


\title{
Effect of Indium addition in U-Zr Metallic Fuel on Lanthanide Migration
}

\begin{abstract}
Advanced fast reactor concepts to achieve ultra-high burnup $(\sim 50 \%)$ require prevention of fuel-cladding chemical interaction (FCCI). Fission product lanthanide accumulation at high burnup is substantial and significantly contributes to FCCI upon migration to the cladding interface. Diffusion barriers are typically used to prevent interaction of the lanthanides with the cladding. A more active method has been proposed which immobilizes the lanthanides through formation of stable compounds with an additive. Theoretical analysis showed that indium, thallium, and antimony are good candidates. Indium was the strongest candidate because of its low reactivity with iron-based cladding alloys.

Characterization of the as-fabricated alloys was performed to determine the effectiveness of the indium addition in forming compounds with lanthanides, represented by cerium. Tests to examine how effectively the dopant prevents lanthanide migration under a thermal gradient were also performed. The results showed that indium effectively prevented cerium migration.
\end{abstract}

Keywords: metallic fuel; fast reactor fuel; FCCI; lanthanide immobilization; out-of-pile test 


\section{Introduction}

Fast reactor design concepts that can achieve ultra-high burnup $(\sim 50$ at $\%)$ without requiring refueling utilizing U-Zr metallic fuel have been proposed [1][2]. One of the primary concerns of this design is fuel pin integrity, which should be maintained by minimizing fuel-cladding-chemical interaction (FCCI) and fuel-swelling-driven fuel cladding mechanical interaction (FCMI). A study addressing the latter issue was reported elsewhere [2]. U-Zr metallic fuel and Fe-based cladding are less compatible than their LWR counterparts $\mathrm{UO}_{2}$ ceramic fuel and Zircaloy cladding. The FCCI issue is more important for the former, particularly because the fuel is planned to achieve high burnup, where fission product accumulation and migration in the fuel are high [4].. Fission product lanthanides (LA) including La, $\mathrm{Pr}$, $\mathrm{Ce}$, and $\mathrm{Nd}$ tend to migrate to the fuel periphery, the kinetics of which depends on the thermal gradient and temperature [4][5], which should be controlled to reduce FCCI. General aspects of FCCI in a metallic fuel element can be found in Ref.[6].

Using the fission yields of LA elements, one can calculate that LA accumulate at a rate approximately 0.4 at $\%$ per at $\%$ burnup in U-10wt $\%$ Zr. Migration of LA to the fuel surface during irradiation is of particular concern because LA are more reactive with the cladding than with fuel constituents. The LAcladding reaction products are known to be harmful to fuel pin performance because of their brittleness and incompatibility with the unreacted cladding. The reacted part of the cladding does not contribute to cladding strength, so the effective cladding wall thickness is reduced. Furthermore, the LA-cladding reaction products and fuel-cladding reaction products that contain LA have lower melting points than the unreacted fuel and cladding, allowing liquefaction to occur in the fuel pin at a much lower temperature $[7][8]$. 
In this paper, as a remedy to prevent FCCI, a chemical modification of U-10Zr alloy fuel was studied. A small amount indium was added to the alloy as a dopant. Cerium was also added to the U-10Zr alloy, representing the LA concentration in a high burnup fuel. The doped alloys were microscopically examined to determine whether the dopant forms a compound with Ce. Two thermal gradient tests were conducted to examine the effect of In addition on LA migration. Samples of U-10Zr-2Ce and U-10Zr2Ce-5In were annealed under thermal gradients $(50-80 \mathrm{~K} / \mathrm{mm})$ for $3-6$ months. The U-10Zr-2Ce sample showed Ce migration, whereas the U-10Zr-2Ce-5In sample showed no Ce migration, implying In prevented Ce migration.

\section{FCCI prevention methods}

From the early metal fuel development era at Argonne National Laboratory (ANL), the most common method to prevent LA-related FCCI was to install a diffusion barrier on the inner surface of the cladding [9]-[11]. The ANL authors adopted various methods to apply a barrier layer on the inner surface of cladding tubes, which were then irradiation tested. Some test results were not promising and were not thought to warrant further studies. The failure rate was unacceptably high due to undetected defects (hairline crack, fissure, hole, etc.) in the barrier. Additionally, it was found that the defective barrier cladding resulted in more severe FCCI than uncoated cladding. Quality assurance to insure the defectfree barrier for this method to be effective was deemed insurmountable and costly.

Interest in the barrier method was revived in recent studies from KAERI, Korea. They examined using out-of-pile diffusion-couple tests the effectiveness of transition metals such as $\mathrm{Zr}, \mathrm{W}, \mathrm{V}$, and $\mathrm{Cr}$ as a diffusion barrier in a form of a thin foil [12], a vapor-deposited layer [13], or an electroplated layer [14]. 
These tests focused on finding the best barrier materials and the method to apply the barrier on the cladding surface. At ANL, atom layer deposition (ALD) method has been proposed to apply a diffusion barrier [14]. The main advantage of this method is the capability of applying a very uniform layer on a complex surface, so defect-free barrier can be produced.

Another way to reduce FCCI is a chemical doping method [4]. One or more elements are added to the fuel to form thermodynamically stable compounds with LA in order to immobilize them. The candidate elements should preferably react with LA over fuel constituents or cladding constituents. The proposed candidate for the dopant method at ANL was indium, from all of the IIIA elements. Idaho National Laboratory (INL) later proposed the same method, but suggested palladium as the most promising dopant [16]-[18].

\section{Requirements for a candidate dopant}

A good dopant candidate should have the following characteristics:

- High affinity with LA to form a stable compound with them

- Low reactivity with fuel constituents (U, Zr) and cladding constituents ( $\mathrm{Fe}, \mathrm{Cr}, \mathrm{Ni})$

- Forming compounds with LA that have low dopant-to-LA ratios so only a small amount of dopant must be added to be effective

- Low neutron absorption cross section for neutron economy

- Have the capability to distribute uniformly and in fine particles in U-10Zr 
Considering the above requirements, IIIA elements such as gallium, indium and thallium appeared to be favorable. These elements generally have low melting points. Thermal neutron absorption cross sections are 193.5, 2.9 and $3.4 \mathrm{~b}$ for $\mathrm{Ga}$, In and $\mathrm{Tl}$, respectively, while fast neutron (> $1 \mathrm{MeV})$ absorption cross sections are $0.0035,0.014$ and $0.0015 \mathrm{~b}$, for $\mathrm{Ga}$, In and Tl, respectively. Therefore, these elements are benign in the neutron economy point of view in a fast spectrum.

Gibbs free energy of formation for a compound $(\Delta \mathrm{G}=\Delta \mathrm{H}-\mathrm{T} \Delta \mathrm{S})$ is the measure of the stability of a compound and spontaneity of the formation reaction. Since enthalpy is the dominant part of Gibbs free energy, particularly at low temperatures, the enthalpy of formation of a compound provides an approximate measure of is the Gibbs free energy relative to other comparable compounds. The more heat released during a reaction, the larger negative value of the enthalpy of formation.

The enthalpies of formation of Ce-Ga, Ce-Tl, and Ce-In alloys as a function of the candidate element concentration were calculated by applying the Miedema model [19], and are shown in Fig. 1 - Fig. 3. Ce was selected to represent the LA based on its close chemical behavior with other LA elements. Also included in the figures are the binary alloys between the fuel constituents and cladding constituent elements with the candidate elements.

In Fig. 1, Ga exhibits negative enthalpies for all binary alloys examined. The negative enthalpies of formation with $\mathrm{U}$ and $\mathrm{Zr}$ suggest that Ga can be consumed by the fuel, particularly by $\mathrm{Zr}$, although $\mathrm{Ga}$ will form compounds with LA. The more significant finding is the negative enthalpies with cladding constituents, indicating that Ga will react with the cladding. 
The thermodynamic assessment for $\mathrm{Tl}$ is shown in Fig. 2. The enthalpies of formation of $\mathrm{Tl}$ with cladding constituents are positive, indicating that $\mathrm{Tl}$ does not spontaneously react with the cladding. However, the large negative enthalpy of formation with $\mathrm{Zr}$ is a disadvantage because it predicts that $\mathrm{Tl}$ will preferentially react with the $\mathrm{Zr}$ in the fuel, rather than LA.

Fig. 3 shows the thermodynamic assessment results for In. The formation enthalpies of Ce-In alloys are the lowest of all the examined reactions, implying that In reacts preferentially with Ce (and the other LA), rather than the fuel or the cladding. The positive enthalpies of formation with cladding constituents, except for the slightly negative values for $\mathrm{Ni}$-In, indicate In will not react with the cladding. From this thermodynamic consideration, In was selected as the most promising element.

According to the Ce-In phase diagram [20], Ce and In can form seven compounds between them. Among these compounds, $\mathrm{Ce}_{5} \mathrm{In}_{4}, \mathrm{Ce}_{3} \mathrm{In}_{5}$, and $\mathrm{CeIn}_{3}$ are formed directly from the melt. In the condition that $\mathrm{Ce}$ is scarce and In is abundant, highly likely in an in-pile irradiation, as Ce atoms are produced by fission events and In is already included in the fuel, $\mathrm{CeIn}_{3}$ is the richest in Ce and is the most favorable compound to form. When Ce concentration increases with burnup, lower In compounds will be form through peritectoid reactions.

\section{Alloy sample characterization}

\subsection{Alloy fabrication}

U-10Zr, U-10Zr-2Ce, U-10Zr-5In, and U-10Zr-2Ce-5In alloys were produced and characterized. Here the number in front of the element stands for weight percent of the element. U-10Zr is the base fuel 
composition at beginning of life (BOL). The U-10Zr-2Ce represents the fuel composition at the end of life (EOL) with a burnup of 10 at $\%$ without a dopant [21]. U-10Zr-5In represents the fuel composition at the BOL with indium as a dopant. U-10Zr-2Ce-5In is to simulate the fuel at 10 at $\%$ burnup with indium as a dopant.

The alloys were prepared by first blending the alloying elements together according to the determined weights. The materials were arc-melted into a button, and melted additionally three times (with an inversion between each melt) to improve homogeneity. The button was then cast into a 9-mm diameter rod, which was further sectioned to create a flat and perpendicular surface. The surface was polished prior to the test.

The sample surfaces were examined using optical microscopy (OM) and scanning electron microscopy (SEM). The group IIIA elements' low melting points were a concern because liquid metal can embrittle the cladding if it exists as pure elemental precipitates. Thus, particular attention was directed at assuring that the dopants were fully incorporated into the U-Zr matrix and did not reside as a separate pure phase.

\subsection{Optical microscopy (OM) characterization of as-alloyed samples}

An OM image of the U-10Zr alloy is shown in Fig. 4(a), and it appears to be quite homogeneous. The dark precipitates are $\alpha-Z r$, while the subtle color variation in the matrix indicates that two phases are present. The slightly darker phase is assumed to be a mixture of $\mathrm{U}$ and $\mathrm{Zr}$, and the brighter phase is $\alpha-\mathrm{U}$ phase. 
An OM image of U-10Zr-2Ce is shown in Fig. 4(b). Compared to the U-10Zr sample, there are far more dark precipitates, which are primarily Ce-rich. Some of the precipitates are Zr-rich, similar toU-10Zr. It is noticeable that the darkest particles are predominantly $\mathrm{UZr}_{2}$, the same as those present in $\mathrm{U}-10 \mathrm{Zr}$. This observation suggests that Ce mixes better with $\mathrm{Zr}$ because of the higher affinity to $\mathrm{Zr}$ than to $\mathrm{U}$.

Fig. 4(c) shows an OM image of the U-10Zr-5In alloy. Compared to the U-10Zr sample, the spherical phases are presumed to be indium-containing precipitates, because these were not present in the U-10Zr alloy shown in Fig. 4(a). Because the LA concentration increases with burnup in a real fuel case, this alloy is the form at beginning of life (BOL).

Characterization of the U-10Zr-2Ce-5In sample was performed. Fig. 4(d) shows an OM image that reveals that the alloy has four phases: $\alpha-U$ and $\delta-U_{Z} r_{2}$ phases in the continuous medium, and a dark precipitate phase that is enclosed in an additional spherical precipitate phase. The darkest phase is a Cerich phase and the spherical phase is an In-rich phase. The observation that the Ce-rich phase is primarily located within the In-rich phase is remarkable, because this implies that In may form compounds with Ce.

\subsection{Scanning electron microscopy (SEM) characterizations and discussion}

Scanning electron microscopy with energy-dispersive x-ray fluorescence spectroscopy (SEM/EDS) was done using a Hitachi S-3000N SEM equipped with a Thermo-Noran Si-Li detector and System Six analysis system. Images were taken using both a secondary electron (SE) detector and a backscatter electron (BSE) detector. Compositional analyses by EDS utilized the "Proza" $(\varphi(\rho z))$ standardless 
analytical correction, assuming a metal sample. The SEM BSE image of the U-10Zr specimen provided in Fig. 5(a) more clearly shows the phases observed in the OM image in Fig. 4(a). The compositional analysis confirmed the assumed composition of the phases observed in the OM image (Table 1).

The addition of $\mathrm{Ce}(2 \mathrm{wt} \%)$ to $\mathrm{U}-10 \mathrm{Zr}$ resulted in the formation of more precipitates, as shown in Fig. 5(b). Composition analysis showed that these dark phases are either Zr-rich or Ce-rich phases (Table 2). The Ce precipitates are formed due to Ce's low solubility in U-Zr alloy[5].

SEM characterization of the U-10Zr-5In alloy (Fig. 5(c)) shows clearly that the spherically shaped phase has a more complex structure than observed with OM (Fig. 4(c)). The composition analysis revealed that the darkest regions are an In-containing phase. As given in Table 3, the EDS analysis also found both a U-rich (point 1 - 3) and a Zr-In phase. No In-rich, or pure In, phases were observed. Although not analyzed with EDS, there were also miniscule particles of a Zr-rich material in the sample, typical for U10Zr based alloys. The Zr-rich (and In-containing) phase was typical for eutectic solidification. The absence of a separate In-rich phase in the sample supports the prospect that indium would not cause liquid metal embrittlement.

Finally, SEM/EDS analysis was conducted on the U-10Zr-2Ce-5In sample (Fig. 5(d) and Table 4). SE imaging was chosen over BSE for this specimen because the high contrast in BSE obscured the phase variation present. The salient phases are the U-rich continuous phase (Point 1), a spherical U-Zr-In phase (Point 2), which contains small dark precipitates composed of a U-Zr-Ce-In phase (Point 3). This unusual geometry might be due to surface tension and/or the order in which the phases solidified from the melt. 
Because Ce is found primarily with In, it appears that Ce efficiently forms compounds with In. Some indium is found with $\mathrm{Zr}$ due to its affinity with $\mathrm{Zr}$, although indium is preferentially attracted to $\mathrm{Ce}$. In a practical irradiation, the In associated with $\mathrm{Zr}$ would likely form compounds with $\mathrm{Ce}$ as the LA concentration increases with burnup.

None of the alloys exhibited high-In (or pure In) phases, of concern because the low melting point of these phases can cause liquid metal embrittlement of the cladding. When In forms compounds with fuel constituents, their melting points are much higher than the pure element. Indium was always found with $\mathrm{Zr}$ in these samples, and in some cases these compounds included Ce, as predicted by the heat of formation of alloy estimation discussed previously.

\section{Thermal gradient tests}

A diffusion test apparatus was designed to examine the effectiveness of indium in immobilizing cerium. Two tests were performed on U-10Zr-2Ce one with and one without indium. For the test with a sample without indium a thermal gradient of $\sim 80 \mathrm{~K} / \mathrm{mm}, 800-400{ }^{\circ} \mathrm{C}$ across a 5 -mm disc was provided for 180 days, simulating that of an in-pile fuel pin. In the test with indium, a thermal gradient of $\sim 50 \mathrm{~K} / \mathrm{mm}$ was established by two surface temperatures of $750{ }^{\circ} \mathrm{C}$ and $500{ }^{\circ} \mathrm{C}$ across the sample and held for 90 days. Because Ce showed migration to the fuel surface in in-pile tests, it was hypothesized that Ce would move to the cooler surface in the test without the indium immobilizer. No Ce migration in the In-added sample would confirm the dopant's intended effectiveness. 


\subsection{Test setup}

A schematic of the sample holder the thermal gradient tests is shown in Fig. 6(a). The alloy sample was heated by two electrical heaters and was placed in a two-part holder. The heating part was stainless steel and the cooling part was copper. A slight clearance was provided to prevent any hard contact, which would result in unwanted heat transport though the sample holder instead of the sample itself. In order to prevent oxidation of the heaters or the samples during testing, the assembly was brazed together after the sample was loaded.

Two thermocouples (TC) were used to measure the sample temperatures, and they were embedded in the sample holder as close as possible to the sample, $\sim 1 \mathrm{~mm}$ from the sample surfaces, to reduce errors in temperature measurement and control. The sample holder was placed on an insulation brick in the sample chamber, which was purged with high-purity (99.9999\%) Ar gas throughout the test (see Fig. 6(b)). The temperatures were controlled to within $5{ }^{\circ} \mathrm{C}$ of the set values during the test. Heat removal by natural convection was sufficient to maintain the desired thermal gradient.

\subsection{Test results}

After the tests, the samples were sectioned axially and the cross-section surface was polished. SEM examination was conducted and EDS was utilized to measure elemental concentrations along the thermal gradient. 
Because $\mathrm{Ce}$ is observed primarily in Ce-rich precipitates, rather than being mixed into the alloy at an atomic level, the EDS line scan method was ineffective in measuring the Ce concentration profile. Instead, the average concentration of Ce in a rectangle approximately $0.25 \mathrm{~mm}$ long and $0.2 \mathrm{~mm}$ wide was measured every $0.2 \mathrm{~mm}$ along the thermal gradient. Sohn et al. [22] used an analogous method to homogenize local peaks due to the presence of multiphase microstructures. The measured concentration profiles for the U-10Zr-2Ce test sample are plotted in Fig. 7. The Zr concentration profile shows the expected enrichment at the hot end from $\mathrm{Zr}$ migrating up the thermal gradient, and the $\mathrm{U}$ profile shows the opposite [23]-[26]. Ce concentration revealed a slight increase toward the hot end and a sharp decrease at the hot end surface. This might be due to Ce migration to the hot end tied to $\mathrm{Zr}$ diffusion, followed by vaporization at the surface.

Fig. 8 shows EDS scan results for U, Zr, Ce, and In in the U-10Zr-2Ce-5In sample. The results show local irregularities due to locally inhomogeneous phases in the sample. The Ce concentration profile shows no appreciable change in concentration along the thermal gradient, suggesting no migration occurred. Indium's concentration profile also appears to be nearly constant. Because In and Ce were together in compounds in the pre-test sample, this confirms that the compounds were preserved during the test and demonstrates that indium immobilized Ce.

\section{Discussion}

Indium was selected as the most promising candidate as a dopant to prevent or reduce LA migration by forming compounds with LA, preventing diffusion. Both the alloy characterization in section 3 and the out-of-pile test results discussed in section 4 support this assumption. 
However, these observations are not enough to conclude that In addition will be effective in-pile, as radiation could substantially affect the behavior. It is known that radiation enhances dissolution of precipitate phases through recoil release of the atoms from the precipitates into the matrix as a result of displacements in collision cascades [27]-[29]. In this mechanism, precipitates can be dissolved by recoil release of the atoms from the precipitates into the matrix as a result of atomic displacements in the collision cascades. A counter-mechanism also exists that drives the dissolved atoms back to the precipitates, or to form new precipitates, by radiation enhanced diffusion (RED).

The image given in Fig. 9 is from an in-pile test of U-Mo dispersion fuel with Si and Mg added to the matrix. The Si-Mg precipitates formed during sample fabrication were dissolved and precipitateremoved zones were formed around the U-Mo surfaces during irradiation [30]. Keiser showed that the freed Si moved to the nearby U-Mo fuel due to Si's high affinity for U [31]. In the present case, indium will be consumed preferentially by Ce. It is anticipated that In initially contained in U-Zr-In compounds will be released from the precipitates due to radiation cascades, freeing it to form more stable In-LA compounds.

Another difference between in-pile irradiation and out-of-pile examinations is LA accumulation with burnup, rather than having a set concentration throughout. For the out-of-pile cases, In and Ce form precipitates. However, for an in-pile irradiation, at BOL no Ce is available for In to form compounds, so it is expected to form a compound with $\mathrm{U}$ and $\mathrm{Zr}$ in fresh fuel (Table 3). As discussed previously, irradiation enhances dissolution of precipitates, potentially releasing the In to form compounds with LA 
as they are formed. In this regard, in-pile irradiation condition may be more favorable the formation of In-Ce compounds.

\section{Conclusions}

In order to control fission product reaction with cladding (FCCI), the migration of fission product LA to cladding needs to be suppressed. A dopant method, in which an element is added in the fuel to chemically immobilize the LA from migration, was studied in this work.

The group III elements in the periodic table of elements were identified as candidate dopants because of their high affinity to lanthanide fission products. A thermodynamic assessment showed that indium appeared to be the strongest dopant candidate, as it would react preferentially with Ce and be least likely to react with the cladding. Ce was used as a representative for the LA as it has similar chemical behavior to the other LA elements.

As-cast alloy sample characterization showed that indium formed compounds with Ce in a U-10Zr-2Ce alloy and that indium did not appear to form high-indium phases, or was not present as a pure element, confirming that indium will not enhance liquid metal embrittlement in cladding. These results are favorable for indium as a candidate dopant.

The dopant alloying tests also showed consistent results with the theoretical predictions. Indium formed compounds with cerium when added to the U-10Zr-2Ce alloy, suggesting its effectiveness as an immobilizer for lanthanide fission products. The proposed alloy is U-10Zr-5In. 
The out-of-pile test of U-10Zr-2Ce under a thermal gradient showed Ce migration, while the In-added alloy out-of-pile test exhibited no sign of Ce migration. Ce was believed to be immobilized by the addition of In, indicating the efficacy of In as a Ce-immobilizer.

The results of this study conclusively showed that the addition of indium to U-10Zr fast reactor fuels has the potential to significantly reduce LA migration, which otherwise is a major cause of cladding failure and provides an obstacle to achieving high burnup. Although only an in-pile test can provide an ultimate evaluation, out-of-pile alloy characterizations and simulation tests show favorable results that warrant further investigation of this method. 


\section{Acknowledgments}

Dr. L. Jamison of Argonne National Laboratory is acknowledged for the review of the manuscript. The submitted manuscript has been created by the UChicago Argonne, LCC as Operator of Argonne National Laboratory under Contract No.DE-AC-02-06CH11357 between UChicago Argonne, LLC and the US Department of Energy.

\section{References}

[1] T.K. Kim, T.A. Taiwo, "Feasibility Study of Ultra-Long Life Fast Reactor Core Concept," Proc. PHYSOR 2010 - Advances in Reactor Physics to Power the Nuclear Renaissance, Pittsburgh, PA, USA, May 9-14, 2010.

[2] A.E. Wright, A.M. Yacout, T.K. Kim, Y.S. Kim et al., Ultra-high Burnup SFR Metallic Fuel: Preliminary Assessment and Plan for Concept Development, Argonne-FCRD-317, 2010.

[3] Yeon Soo Kim, Y.S. Choo, H.J. Ryu, A.M. Yacout, G.L. Hofman, Trans. Am. Nucl. Soc. 103 (2010) 289.

[4] Yeon Soo Kim, G.L. Hofman, A.M. Yacout, J. Nucl. Mater. 392 (2009) 164.

[5] Yeon Soo Kim, T. Wiencek, E. O’Hare et al., Nucl. Eng. Design, submitted, 2016.

[6] D.D. Keiser, Jr., Metal fuel-cladding interaction, in: R.J.M. Konings (ed.), Comprehensive Nuclear Materials, V. 3, pp. 423-441, Elsevier, 2012.

[7] H. Tsai, The 1990 International Fast Reactor Safety Meeting, Snowbird, UT, USA, Am. Nucl. Soc., Aug. 12-16, 1990.

[8] A.B. Cohen, H. Tsai, L.A. Neimark, J. Nucl. Mater. 204 (1993) 244-251.

[9] W.N. Beck, R.J. Fousek, and J.H. Kittel, Argonne-7388, Argonne National Laboratory, 1968.

[10] A.B. Cohen, T.C. Wiencek, H. Tsai, CONF-9405123-1, Argonne National Laboratory, 1994.

[11] D.C. Crawford, C.E. Lahm, H. Tsai, R.G. Pahl, J. Nucl. Mater. 204 (1994) 157.

[12] H.J. Ryu, B.O. Lee, S.J. Oh, J.H. Kim, C.B. Lee, J. Nucl. Mater. 392 (2009) 206.

[13] J.H. Kim, H.J. Ryu, J.H. Baek, S.J. Oh, B.O. Lee, C.B. Lee, Y.S. Yoon, J. Nucl. Mater. 394 (2009) 144.

[14] S.W. Yang, H.J. Ryu, J.H. Kim, B.O. Lee, C.B. Lee, J. Nucl. Mater. 401 (2010) 98. 
[15] S. Bhattacharya, private communication, Argonne National Laboratory, 2014.

[16] R.D. Mariani, D.L. Porter, T.P. O’Halleran, S.L. Hayes, J.R. Kennedy, J. Nucl. Mater. 419 (2011) 263.

[17] G.W. Egeland, R.D. Mariani, T. Hartmann, D.L. Porter, S.L. Hayes, J.R. Kennedy, J. Nucl. Mater. 432 (2013) 539.

[18] G.W. Egeland, R.D. Mariani, T. Hartmann, D.L. Porter, S.L. Hayes, J.R. Kennedy, J. Nucl. Mater. 440 (2013) 178.

[19] F.R. de Boer et al., Cohesion in metals - Transition metal alloys, North-Holland, 1998.

[20] H. Okamoto, Ce-In (Cerium-Indium), Binary Alloy Phase Diagrams, II Ed., Ed. T.B. Massalski, Vol. 2, 1990, p 1071-1073.

[21] Yeon Soo Kim, T. Wiencek, E. O’Hare, J. Fortner, Global, 2013, Salt Lake City, UT, Sept. 29Oct. 3, 2013.

[22] Y.H. Sohn, M.A. Dayananda, G.L. Hofman, R.V. Strain, S.L. Hayes, J. Nucl. Mater. 279 (2000) 317.

[23] T. Ogawa and T. Iwai, J. Less-Common Metals, 175 (1991) 59.

[24] G.L. Hofman, S.L. Hayes and M.C. Petri, J. Nucl. Mater. 227 (1996) 277.

[25] M. Ishida, T. Ogata and M. Kinoshita, Nucl. Technol., 104 (1993) 37.

[26] Yeon Soo Kim, S.L. Hayes, G.L. Hofman, A.M. Yacout, J. Nucl. Mater. 359 (2006) 17.

[27] R.S. Nelson, J.A. Hudson, D.J. Mazey, J. Nucl. Mater. 44 (1972) 318-330.

[28] P. Wilkes, J. Nucl. Mater. 83 (1979) 166-175.

[29] G.S. Was, Fundamentals of Radiation Materials Science, Springer, 2007.

[30] Yeon Soo Kim, G. Hofman, A. Robinson, D. Wachs, Nucl. Technol. 184 (2013) 42.

[31] D. Keiser et al., J. Nucl. Mater. 425 (2012) 156. 


\section{$\underline{\text { List of table captions }}$}

Table 1 Composition analysis result of the U-10Zr sample. The analysis points are marked in Fig. 5(a).

Table 2 Composition analysis result of the U-10Zr-2Ce sample. The analysis points are marked in Fig. 5(b).

Table 3 Composition analysis result of the U-10Zr-5In sample. The analysis points are marked in Fig. 5(c).

Table 4 Composition analysis result of the U-10Zr-2Ce-5In sample. The analysis points are marked in Fig. 5(d).

\section{$\underline{\text { List of figure captions }}$}

Fig. 1 Enthalpies of formation of gallium-compounds with $\mathrm{Ce}$ and fuel and cladding constituents.

Fig. 2 Enthalpies of formation of thallium-compounds with Ce and fuel and cladding constituents.

Fig. 3 Enthalpies of formation of indium-compounds with Ce and fuel and cladding constituents.

Fig. 4 Optical microscopy (OM) images of the alloy samples.

Fig. 5 Scanning Electron Microscopy (SEM) images of the alloy samples.

Fig. 6 Schematics showing the test design.

Fig. 7 EDS concentration analysis results along the thermal gradient of the U-10Zr-2Ce sample.

Fig. 8 EDS concentration analysis results along the thermal gradient of the U-10Zr-2Ce-5In sample.

Fig. 9 OM image showing the Si-precipitate-depletion zones around the U-Mo fuel particles embedded in the Al matrix. The sample was irradiated to a fission density of $3.4 \times 10^{21} \mathrm{f} / \mathrm{cm}^{3}$ at $115^{\circ} \mathrm{C}$ for 90 EFPD. The dark small particles are the Si-precipitates in the Al matrix. The fission fragment range in $\mathrm{Al}$ is $\sim 12 \mu \mathrm{m}$. 
Table 1 Composition analysis result of the U-10Zr sample. The analysis points are marked in Fig. 5(a).

\begin{tabular}{ccc}
\hline Analysis location & $\begin{array}{c}\mathbf{U} \\
(\mathbf{a t} \%)\end{array}$ & $\begin{array}{c}\mathbf{Z r} \\
(\mathbf{a t} \%)\end{array}$ \\
\hline Point 1 & 87 & 13 \\
Point 2 & 69 & 31 \\
Point 3 & 8 & 92 \\
\hline
\end{tabular}

Table 2 Composition analysis result of the $\mathrm{U}-10 \mathrm{Zr}-2 \mathrm{Ce}$ sample. The analysis points are marked in Fig. 5(b).

\begin{tabular}{cccc}
\hline Analysis location & $\begin{array}{c}\mathbf{U} \\
(\mathbf{a t} \%)\end{array}$ & $\begin{array}{c}\mathbf{Z r} \\
(\mathbf{a t} \%)\end{array}$ & $\begin{array}{c}\mathbf{C e} \\
(\mathbf{a t} \%)\end{array}$ \\
\hline Point 1 & 82 & 17 & 1 \\
Point 2 & 63 & 35 & 2 \\
Point 3 & 5 & 92 & 3 \\
Point 4 & 4 & 2 & 94 \\
\hline
\end{tabular}


Table 3 Composition analysis result of the U-10Zr-5In sample. The analysis points are marked in Fig. 5(c).

\begin{tabular}{cccc}
\hline Analysis location & $\begin{array}{c}\mathbf{U} \\
(\mathbf{a t} \%)\end{array}$ & $\begin{array}{c}\mathbf{Z r} \\
(\mathbf{a t} \%)\end{array}$ & $\begin{array}{c}\mathbf{I n} \\
(\mathbf{a t} \%)\end{array}$ \\
\hline Point 1 & 95 & 4 & 1 \\
Point 2 & 82 & 15 & 3 \\
Point 3 & 85 & 12 & 3 \\
Point 4 & 11 & 64 & 25 \\
\hline
\end{tabular}

Table 4 Composition analysis result of the U-10Zr-2Ce-5In sample. The analysis points are marked in Fig. 5(d).

\begin{tabular}{ccccc}
\hline Analysis location & $\begin{array}{c}\mathbf{U} \\
(\mathbf{a t} \%)\end{array}$ & $\begin{array}{c}\mathbf{Z r} \\
(\mathbf{a t} \%)\end{array}$ & $\begin{array}{c}\mathbf{C e} \\
(\mathbf{a t} \%)\end{array}$ & $\begin{array}{c}\text { In } \\
(\mathbf{a t} \%)\end{array}$ \\
\hline Point 1 & 89 & 7 & 0 & 4 \\
Point 2 & 29 & 51 & 1 & 19 \\
Point 3 & 18 & 39 & 16 & 27 \\
\hline
\end{tabular}




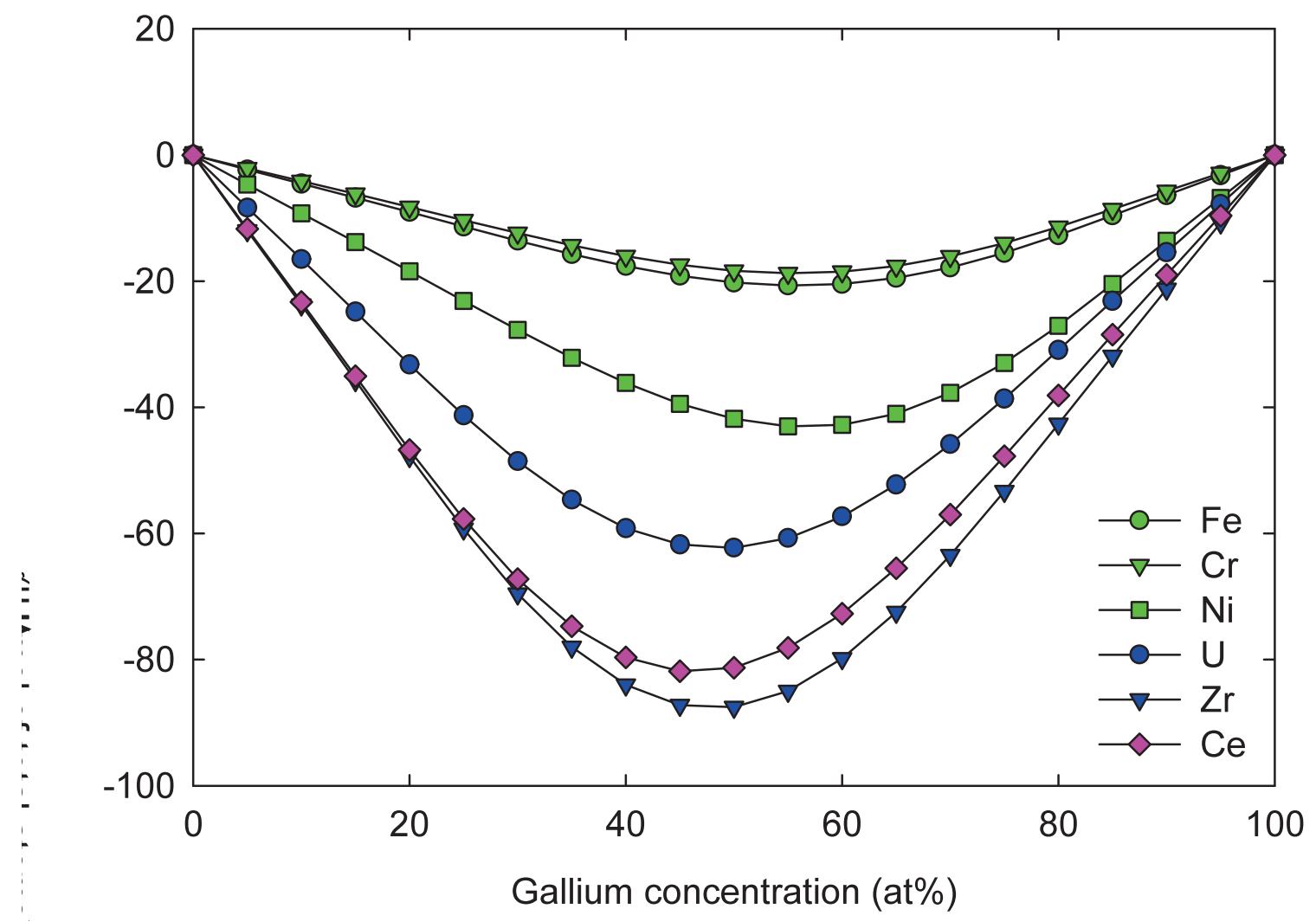

Fig. 1 Enthalpies of formation of gallium-compounds with Ce and fuel and cladding constituents. 


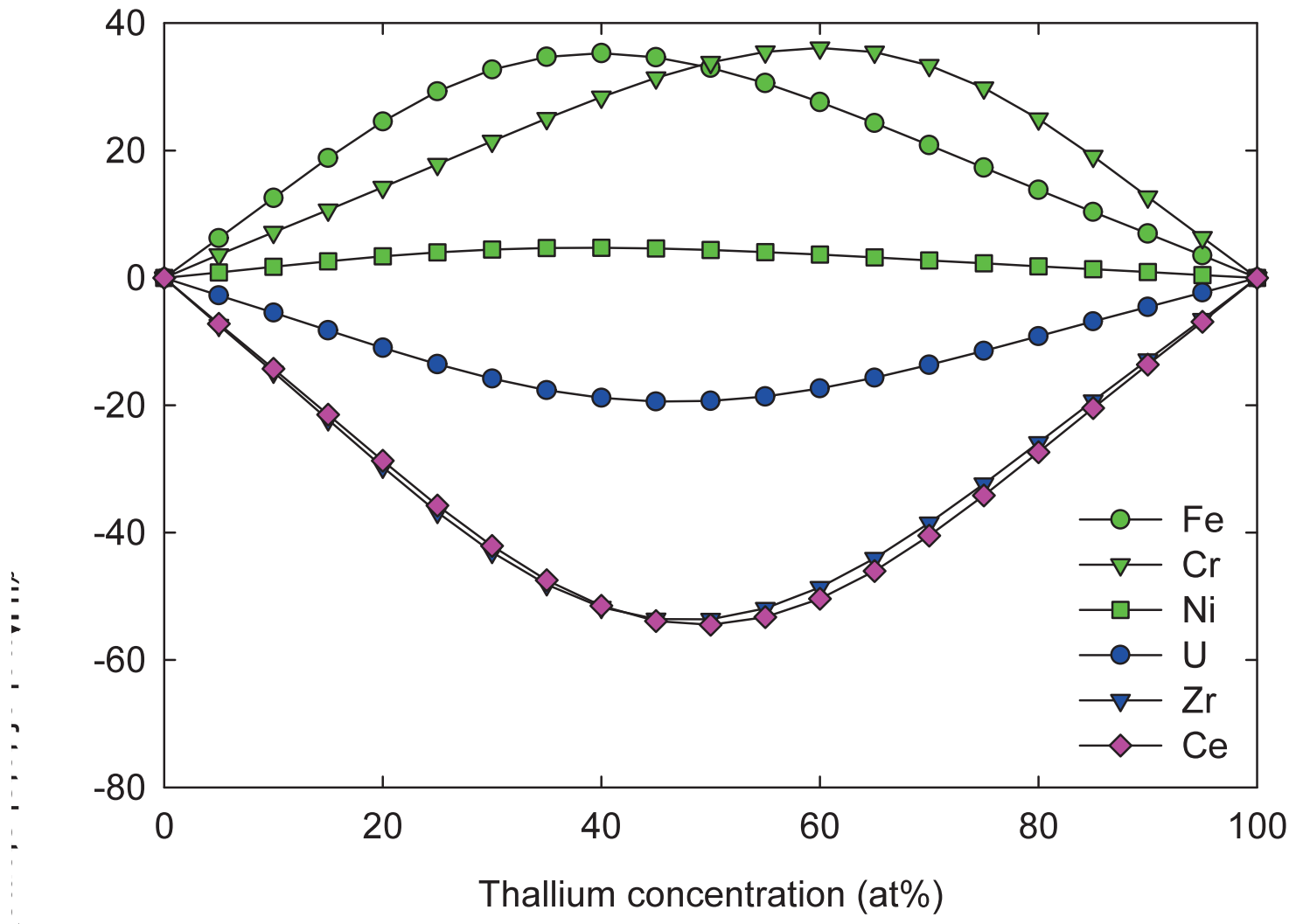

Fig. 2 Enthalpies of formation of thallium-compounds with $\mathrm{Ce}$ and fuel and cladding constituents. 


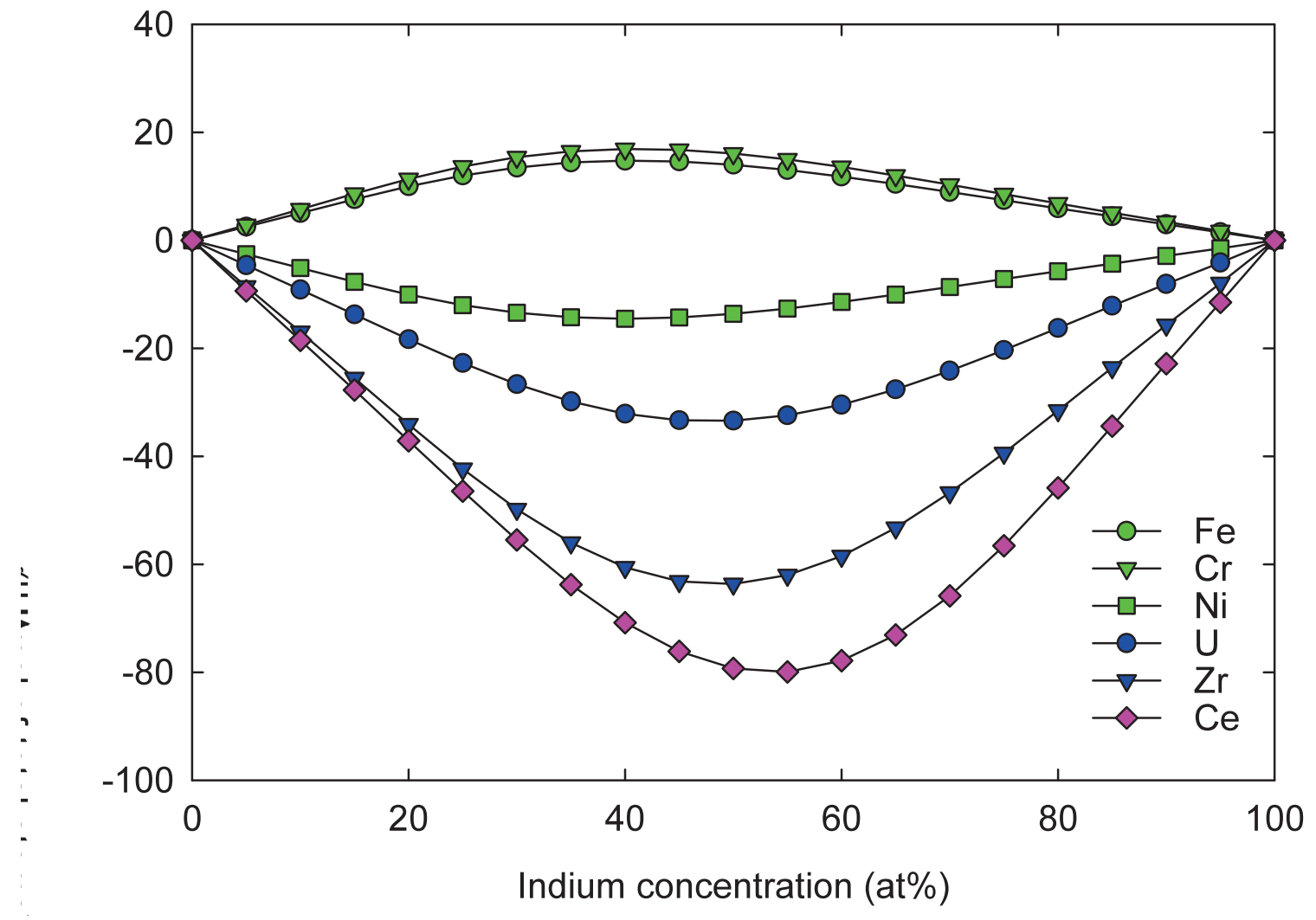

Fig. 3 Enthalpies of formation of indium-compounds with Ce and fuel and cladding constituents. 


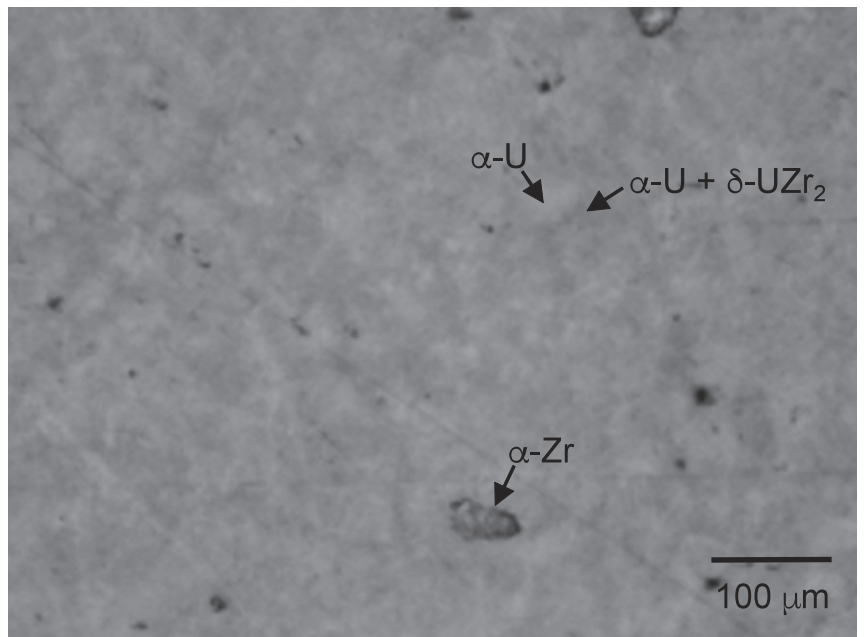

(a) $\mathrm{U}-10 \mathrm{Zr}$

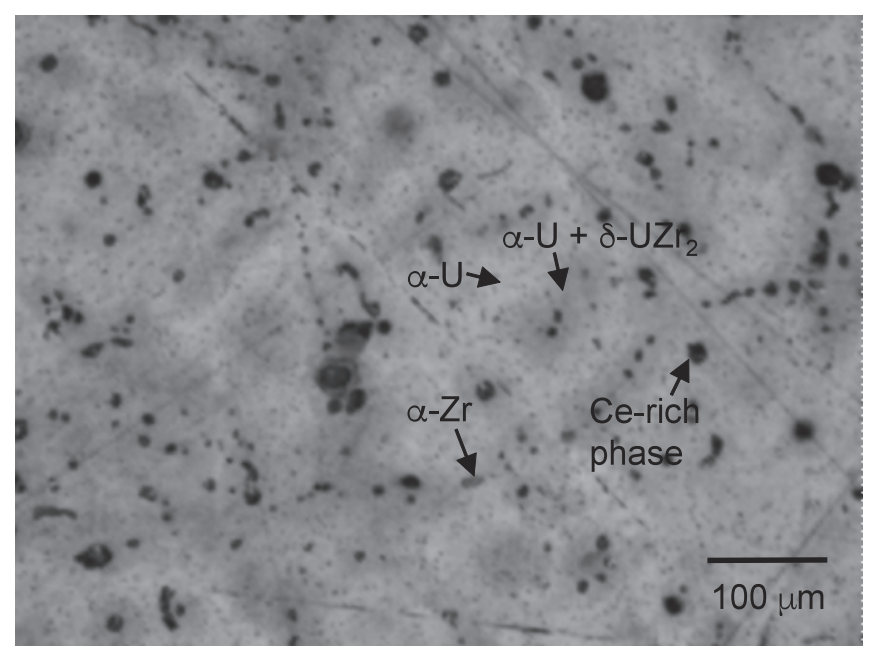

(b) U-10Zr-2Ce

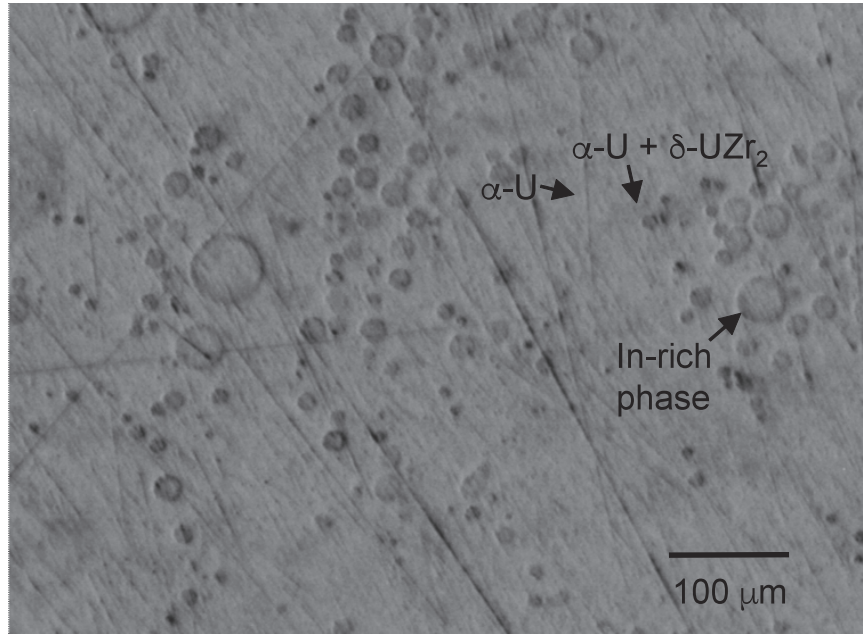

(c) U-10Zr-5In

Figure 4. Optical microscopy (OM) images of the alloy samples 


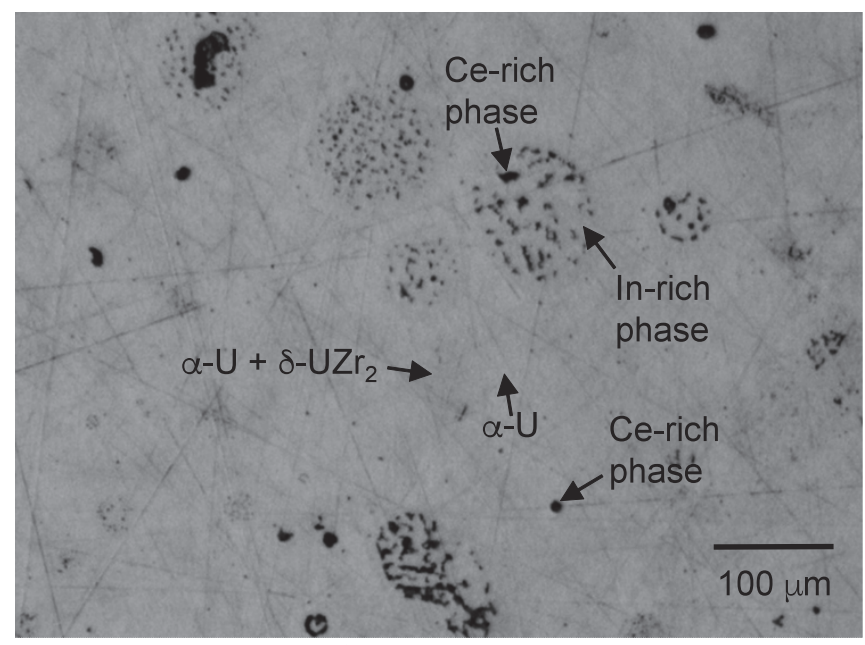

(d) U-10Zr-2Ce-5In

Fig. 4 Optical microscopy (OM) images of the alloy samples. 


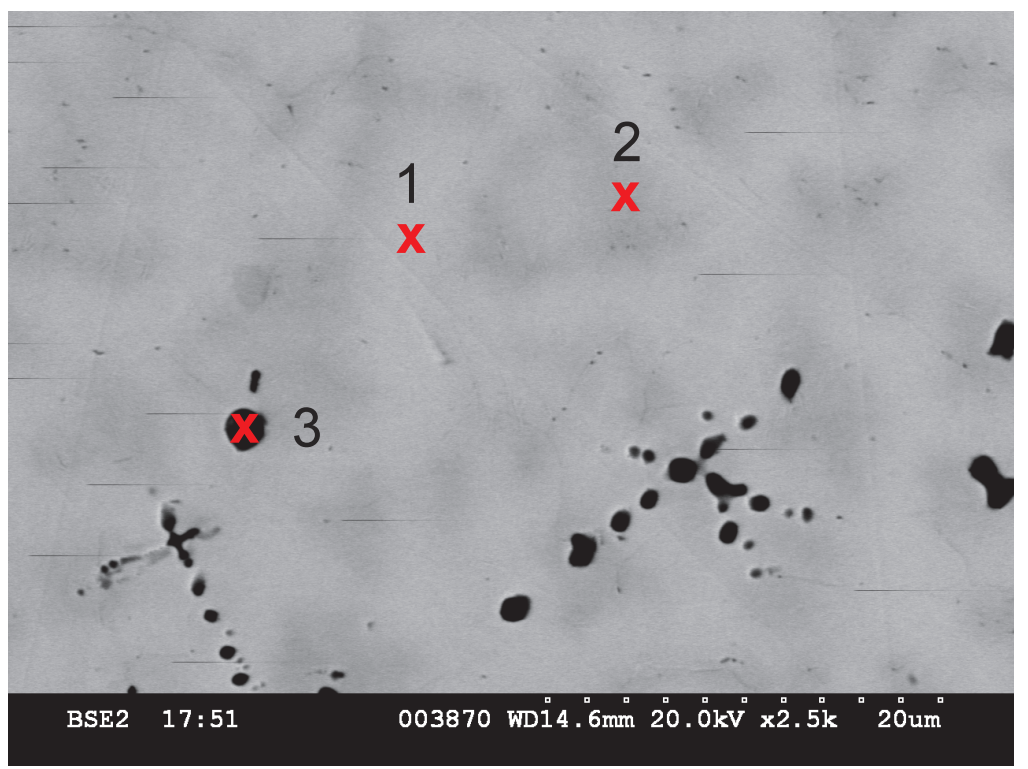

(a) SEM BSE image of U-10Zr. The composition analysis points are marked with numbers.

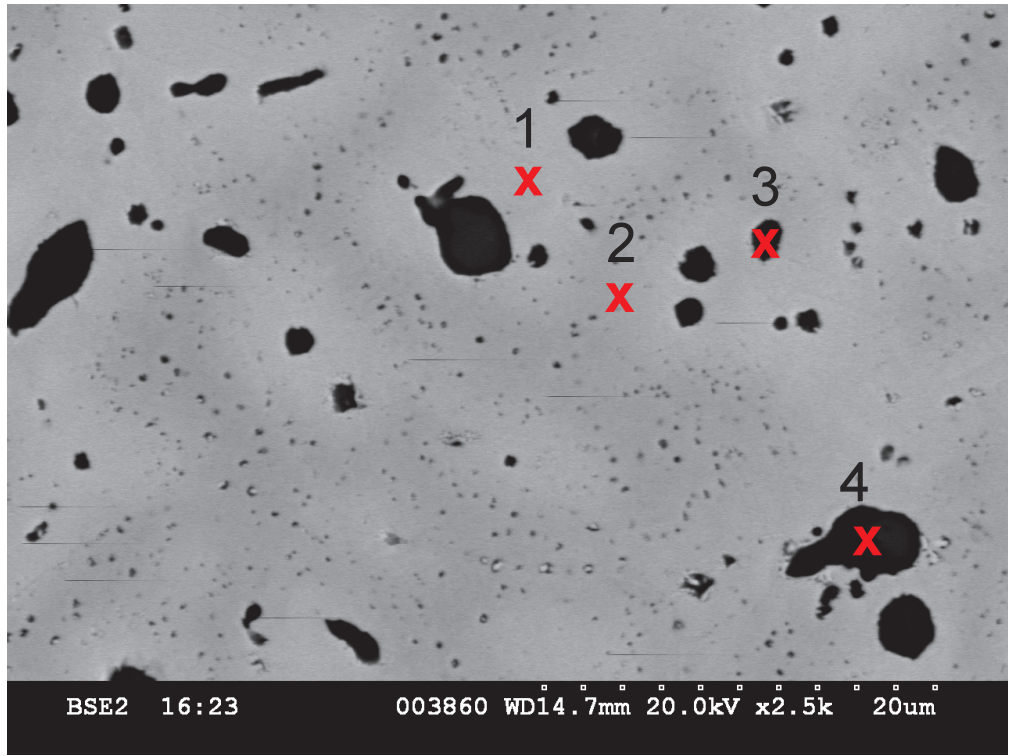

(b) SEM BSE image of U-10Zr-2Ce. The composition analysis points are marked with numbers.

Figure 5. Scanning Electron Microscopy (SEM) images of the alloy samples. 


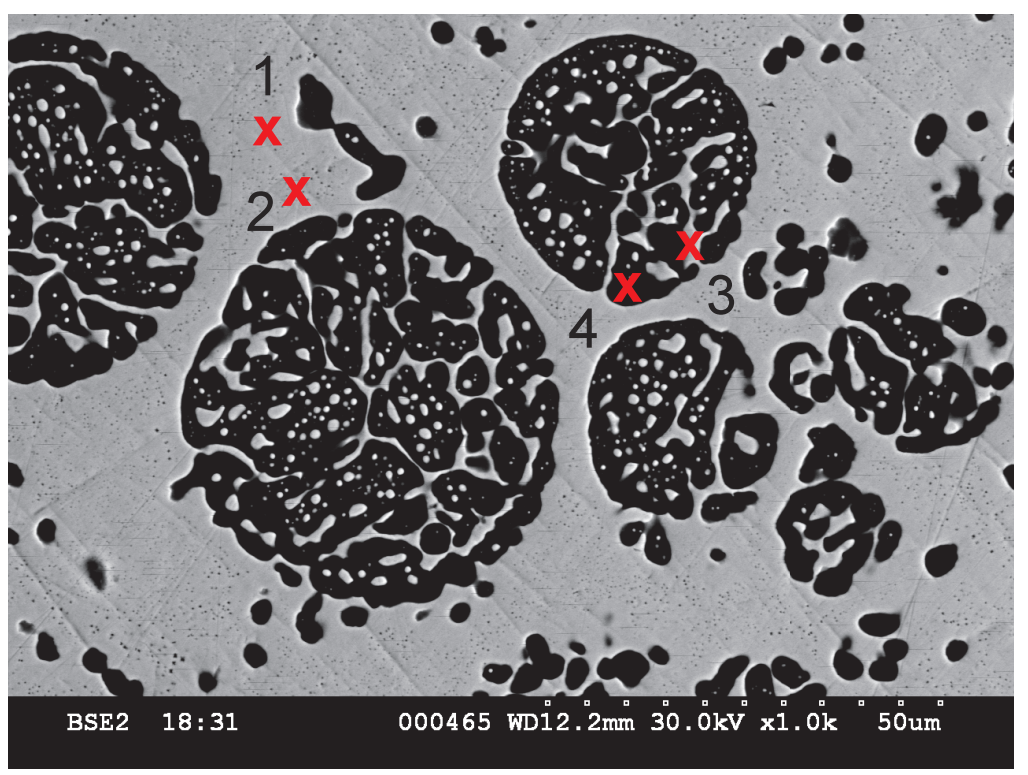

(c) SEM BSE image of U-10Zr-5In. The composition analysis points are marked with numbers.

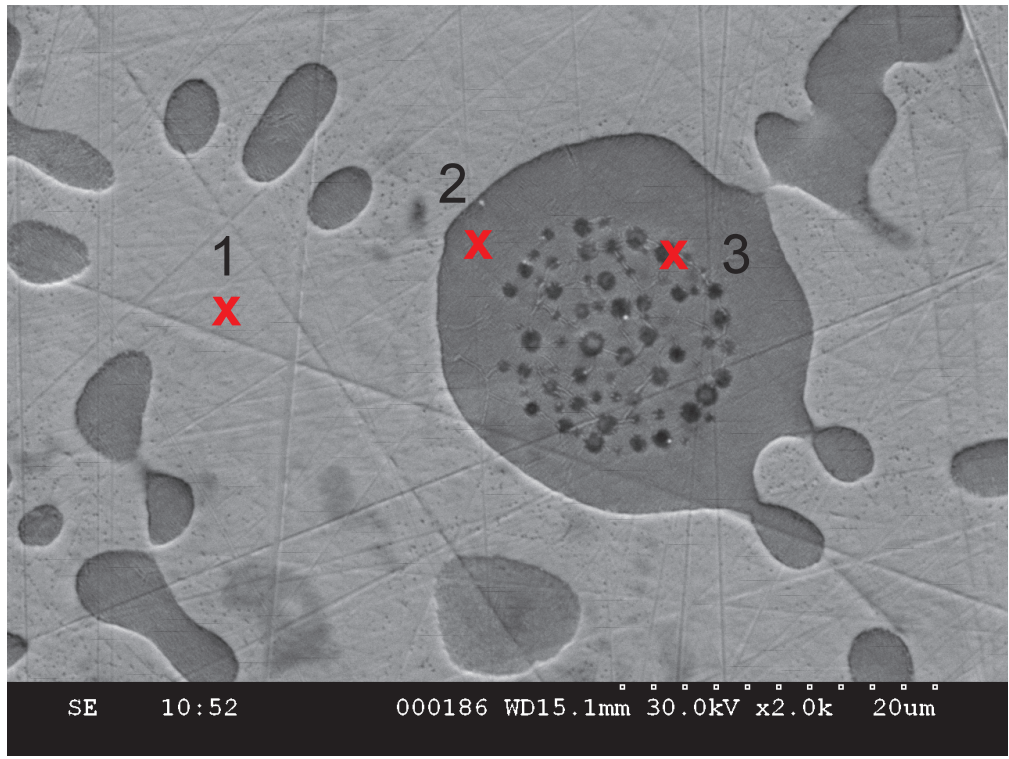

(d) SEM SE image of U-10Zr-2Ce-5In. The composition analysis points are marked with numbers.

Fig. 5 Scanning Electron Microscopy (SEM) images of the alloy samples. 


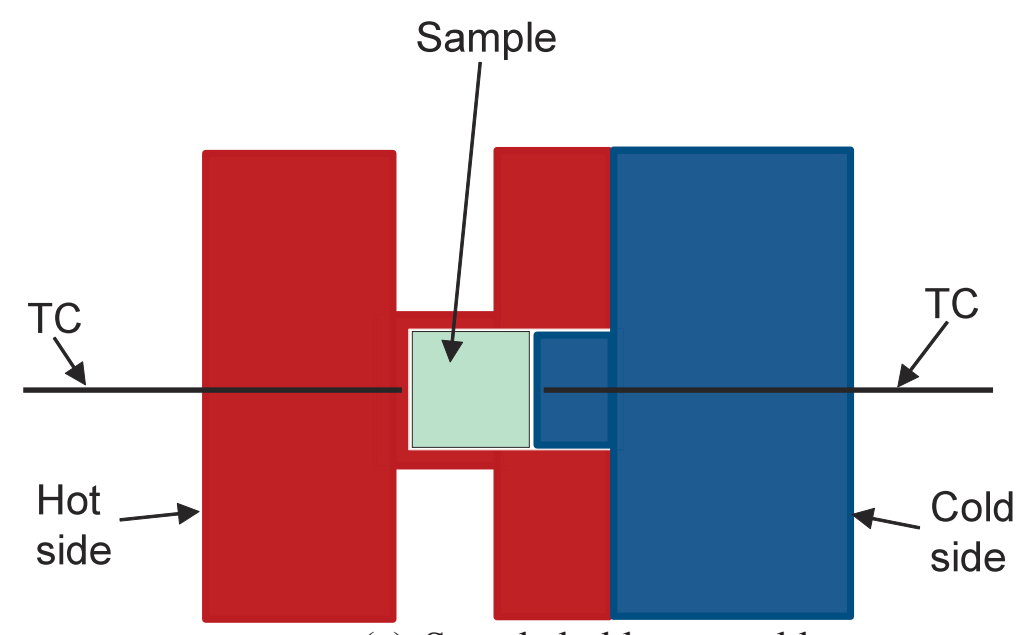

(a) Sample holder assembly

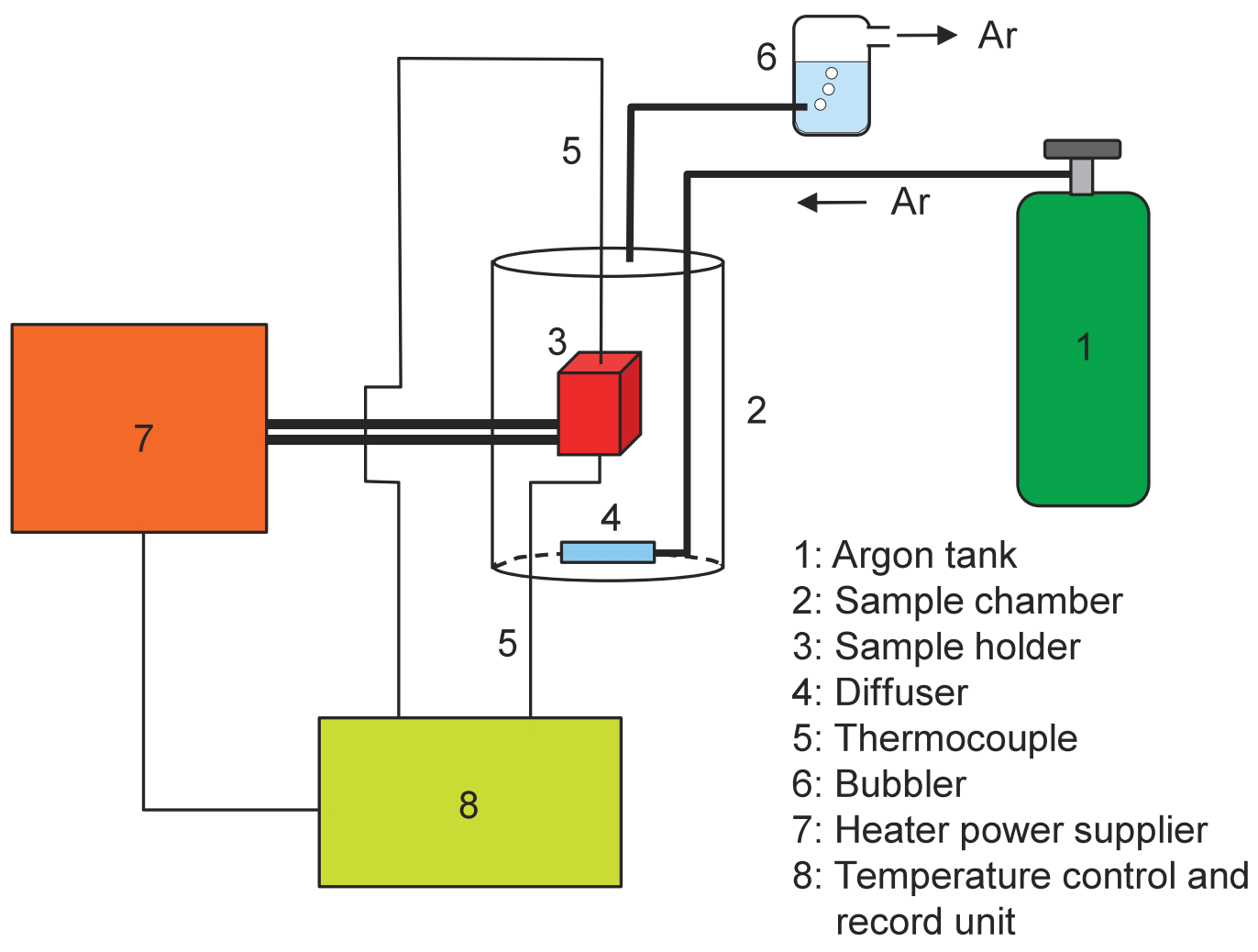

(b) Experimental setup

Fig. 6 Schematics showing the test design. 


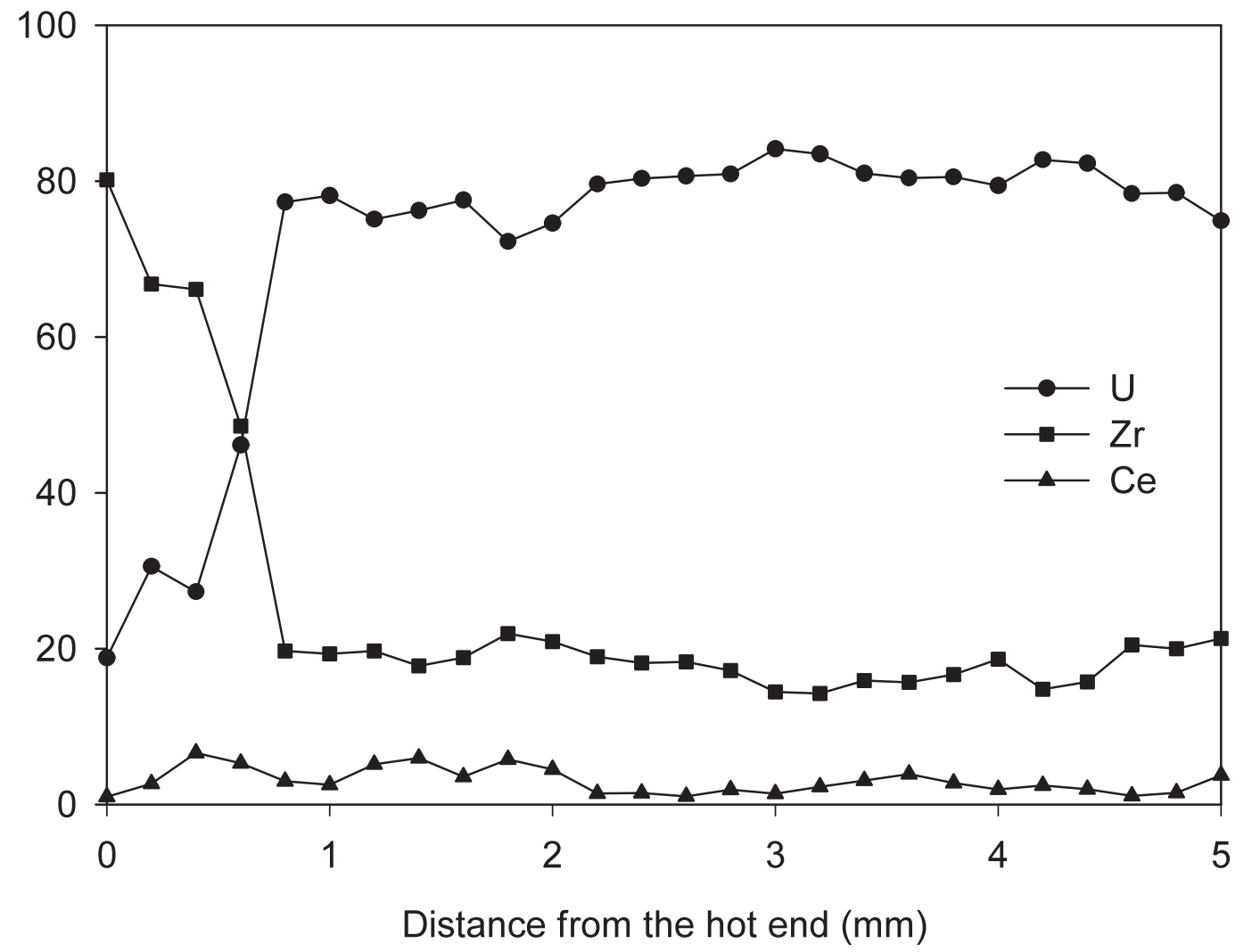

Fig. 7 EDS concentration analysis results along the thermal gradient of the U-10Zr-2Ce sample. 


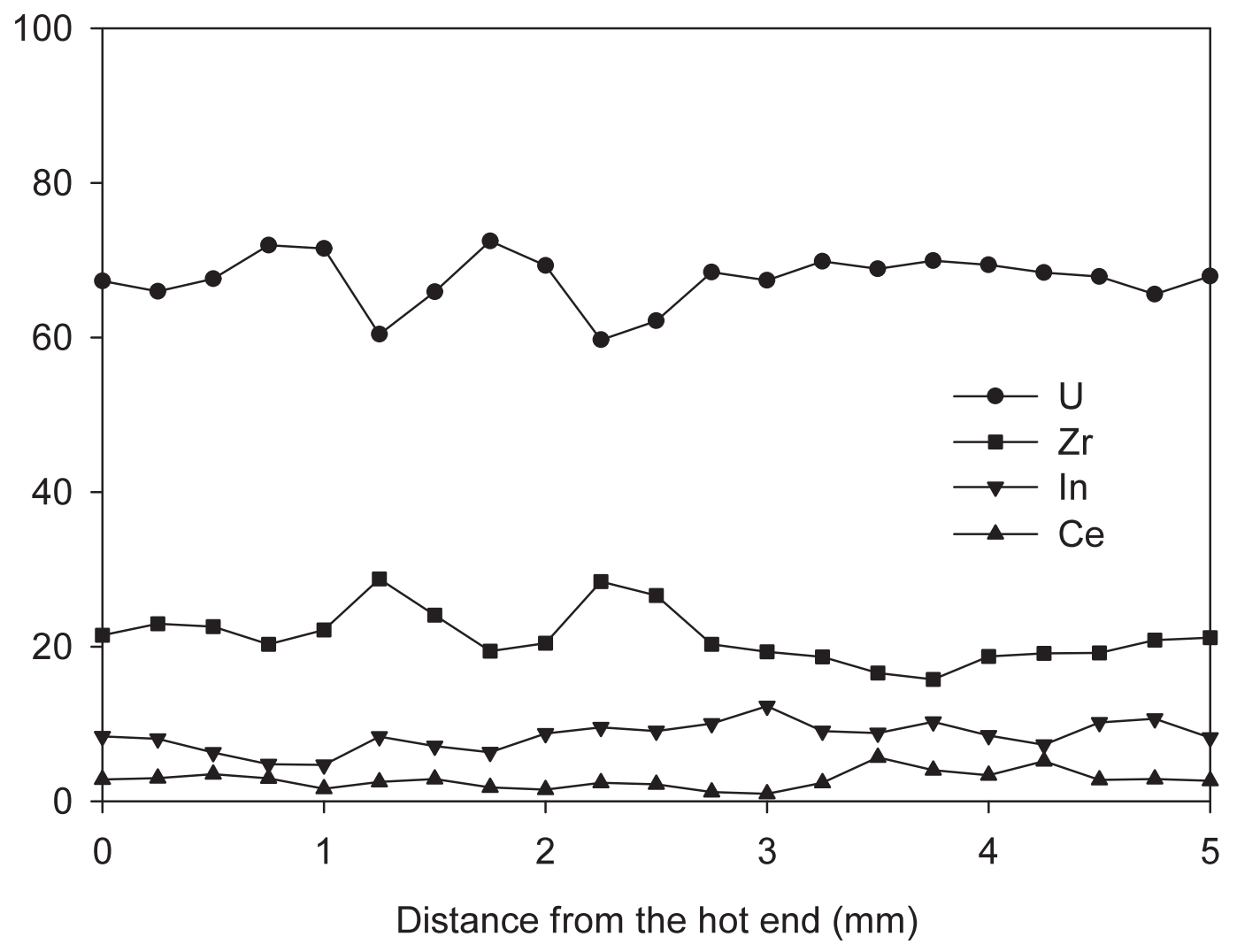

Fig. 8 EDS concentration analysis results along the thermal gradient of the U-10Zr-2Ce5In sample. 


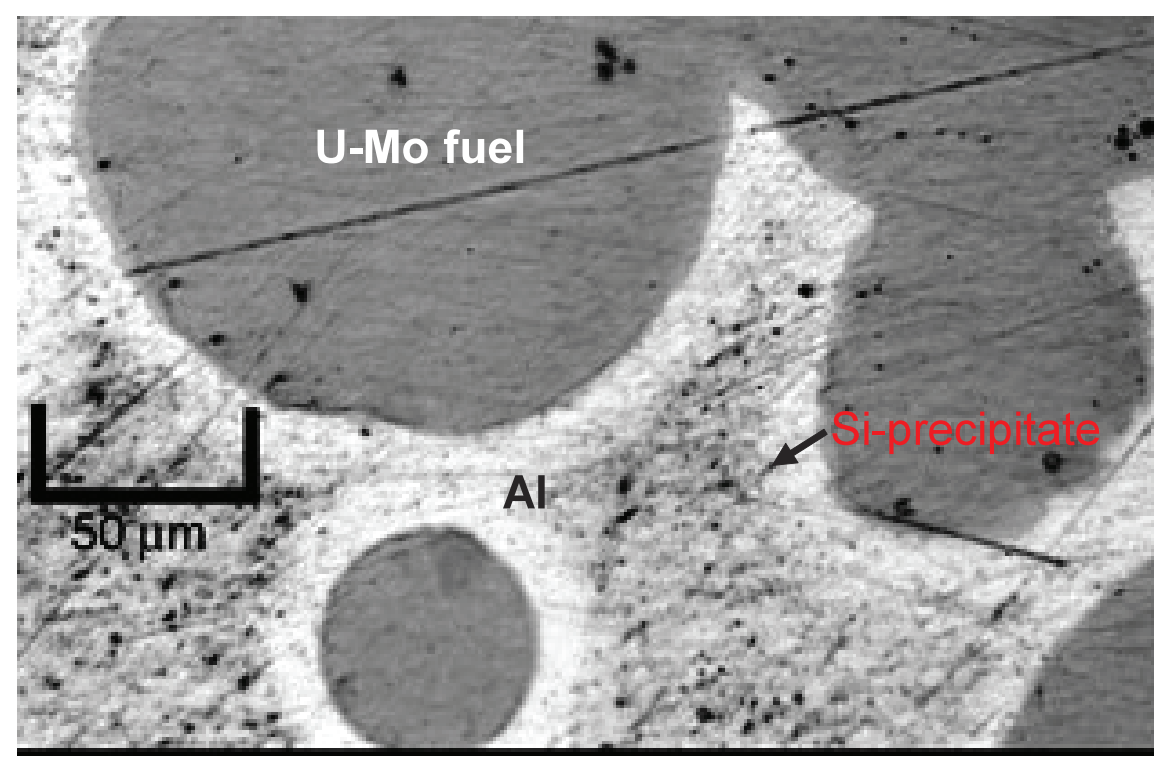

Fig. 9 OM image showing the Si-precipitate-depletion zones around the U-Mo fuel particles embedded in the Al matrix. The sample was irradiated to a fission density of $3.4 \times 10^{21} \mathrm{f} / \mathrm{cm}^{3}$ at $115^{\circ} \mathrm{C}$ for 90 EFPD. The dark small particles are the Siprecipitates in the $\mathrm{Al}$ matrix. The fission fragment range in $\mathrm{Al}$ is $\sim 12 \mu \mathrm{m}$. 